
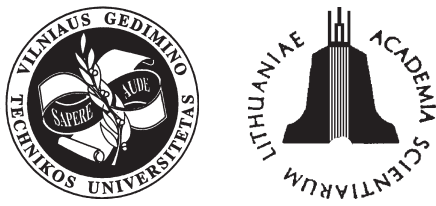

\title{
THE MODEL OF ALLOTMENTS BOOKING IN A SHUTTLE TRAIN
}

\author{
Andrius Jaržemskis \\ Vilnius Gediminas Technical University, Transport Research Institute, \\ Plytines g. 27, LT-10105 Vilnius-16, Lithuania. Tel.: (+370 5) 26997 14; \\ E-mail:andrius.j@omni.lt
}

Received 5 July 2005 ; accepted 30 September 2005

\begin{abstract}
The article presents the model of allotments booking for trucks or containers in shuttle trains. This model could be used in practice if railway companies sale free allotments in rolling stock for forwarders. This paper proposes a stochastic dynamic programming model of a short-term capacity planning model for necessary allotment. The allotment could be acquired by freight forwarders many months ahead on a contract basis, but usually the forecast demand is unreliable. The preplanning of allotment for transport units is needed when the date draws nearer to the train departure time. The presented model evaluates the optimal cost policy based on economic trade-off between the cost of backlogged shipment and the cost of acquiring additional allotment.
\end{abstract}

Keywords: allotment, stochastic model, shuttle train, transport unit.

\section{Introduction}

A shuttle train is new technological implementation for freight transportation in East Europe [1]. In recent years there has been an increasing trend of freight transportation by a shuttle train KlaipedaOdesa, in July 2005 Klaipeda-Moscow starts.

The relevance of this article is based on the idea of shuttle train allotments for forwarders.

The purpose of this article is to device a stochastic dynamic programming model which is necessary for planning of allotments in a shuttle train for trucks or containers.

Shuttle trains (ST) concept could be more efficient if railway companies provided transportation

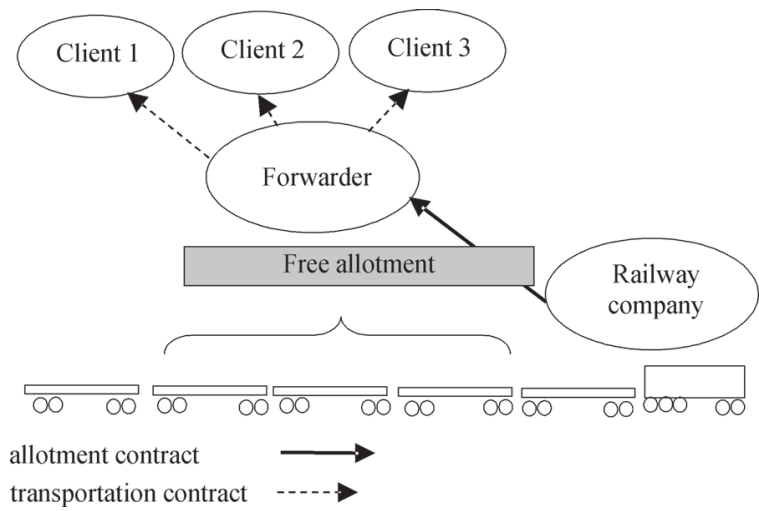

Allotments idea in a shuttle trains concept service sale function to forwarders. This idea is near to freight transportation by air principles.

The current forwarding process in a shuttle trains concept makes capacity planning difficult for forwarders and railway companies as well. Due to high capital investment regular train schedules and rolling stock capacity are planned and fixed by a railway company to last for several months. In an allotment model freight forwarders could acquire free allotment in ST on a long-term contract basis. In such a contract the capacity is planned to the exact amount on each specific dated departure. Therefore, a long-term booking process requires freight forwarders to commit to allotment ahead of time which implies that the shippers must give the freight forwarders their forecasted order fulfillment plans.

There has been very limited work done in capacity planning models for train shipment

Generally, to some degree, our work is similar to Chew, Huang, Johnson, Nemhauser, Sokol and Leong presented model cargo space booking [2]. Instead of deciding how much free space is necessary for cargo in an airplane state what allotment is necessary in rolling stock of a shuttle train for trucks and containers.

The similar principles of modeling could be found in Raman work [3], where the problem is characterized by a finite selling season and uncertain demand, and there may be several periods to produce or purchase the items to be sold during the selling season.

Other way to solve our scientific problem is rout- 
ing and scheduling and fleet sizing too. Effective local search algorithms for routing and scheduling problems with general time-window constraints are presented in Ibaraki, Imahori, Kubo, Masuda, Uno and Yagiura work [4]. These algorithms and Hartman and Wilson [5] model of fleet sizing could be successfully adapted together with my presented model.

Since the amount of TU to be shipped depends on actual rather than planned orders, this process can create high fluctuation in the allotment requirement.

The situation becomes critical when there is a sudden build-up of large orders that creates a shortage in capacity. Then either the order fulfillment has to be delayed which would result in the deterioration of service, or shippers have to send a transport unit by road and significantly increase the logistics cost.

Monthly or quarterly total demands can be forecasted meaningfully, but not daily demands. Usually, the freight forwarders must pay for any unused allotment in a shuttle train. Furthermore, anticipating any sudden rise in demand, the freight forwarders may also need to acquire additional allotment at a higher cost to maintain the service level promised to shippers. This paper develops a decision planning model to determine the optimal short-term allotment to acquire given the long-term contract allotment. The economic analysis trades off between the cost of backlogged shipment and the cost of acquiring additional allotment.

The decision planning model is a multi-period newsvendor type problem and is formulated as a stochastic dynamic programming model where the state variables are the backlogged orders and the decision variables are the additional allotment to be acquired. At every stage the decision maker may increase capacity for the next departures at extra cost to offset the future penalty cost for having backlogged shipments. The decision depends on the current state that is observed, the forecast of the next incoming shipment and the available long-term contract allotment. In practice, there is a limit on how much additional allotment can be acquired. A reasonable assumption is to model the cost of acquiring additional allotment by a convex increasing function with respect to the additional allotment acquired. Any allotment allocated in the period that is not used cannot be returned.

\section{A model of allotments booking and dynamic pro- gramming formulation}

The purchasing of allotment in a shuttle train for a specified dated departure takes place in two time periods. The first round of purchasing occurs in the bidding season when the railway companies sell allotment to freight forwarders on a long-term contract basis. This could occur six months in advance and freight forwarders could commit allotment on each specific dated departure far ahead of time. The second round of purchasing occurs when the dated departure draws nearer. The demand forecast becomes more accurate and the freight forwarders may decide to buy additional allotment. Very often this ad-hoc allotment could be acquired with a higher price than the normal charge for the long-term allocated allotment. The forecasted daily demands will be only meaningful for the next two or three days. Given these forecasted demands and the long-term allotment allocation, a short-term capacity planning model determines the additional allotment required over the next two or three days so that the overall cost is minimized. Within the planning horizon period there are points which define the cut-off time of a particular departure. At these points in time all transport units to be sent out on the next departing shuttle train must have been received. Any allotment remaining that is not used cannot be returned. Immediately after the cutoff time, the decision maker has to decide whether to acquire additional allotment for the next departure. This depends on the amount of backlogged cargo remaining as well as the transport unit that is forecasted to be received between this time and the next immediate cut-off time. Since there may be more than one departure on a given day, we represent our stages as the cut-off times of particular departures. We restrict our problem to a six-stage problem representing a period of three days, assuming that there are two departures a day and the day-by-day demand forecasts are meaningful only for the next three days. The problem can be formulated as a stochastic program which is stated as follows: Consider $i$-th stage. Given the amount of backlogged shipment right after the cutoff time, the long-term allotment allocation and the forecasted demand which is characterized by a probability distribution function, the decision is to acquire allotment $R i$ for the next departure so that the expected cost to the end of the planning horizon is minimized.

Task 1

$$
\begin{aligned}
& Q_{i}\left(B_{i-1}\right)=C \cdot B_{i-1}+\min _{R_{i}: R_{i} \geq 0}\left\{\left(R_{1}\right)+\right. \\
& \left.E_{W_{i}} \max \left(Q_{i+1} \cdot\left(W_{i}-\left(A_{i}-B_{i-1}+R_{i}\right)\right)\right)\right\}, \\
& i=1, \ldots, 6, \\
& Q_{7}\left(B_{6}\right)=Y\left(B_{6}\right),
\end{aligned}
$$

here $Q_{i}\left(A_{i-1}\right)$ - optimal expected cost from stage $i$ to the end of the planning horizon given the current entering state $\mathrm{B}_{i-1} ; C$-backlog cost per unit per period of shipment not shipped out; $B_{i-1}-$ amount of 
backlogged cargo at stage $i$ for $i$-th shuttle train departure; $W i$-demand at stage $i$ which is a random variable that follows a given probability distribution function; A $i$ - amount of long-term allotment allocated for $i$ th ST departure; $R i$-amount of ad-hoc allotment acquired for $i$-th departure; $E_{\mathrm{W}}-$ is the expectation over the forecasted demand distribution; $Z(r)$ - convex increasing cost function of acquiring $r$ units of allotment; $Y(r)$ - convex increasing cost function of $r$ units of allotment being backlogged at the end of the planning horizon.

Let's make several assumptions. There is a given amount of long-term allotment allocated for each shuttle train departure. The forecasted demand in every stage is distributed independently. There may be transport unit backlogged from previous departure. The penalty cost is proportional to the number of periods and the size of the backlogged shipment. Adhoc allotment cannot be acquired with certainty. To model this we use a convex increasing cost function to capture the uncertainty. The penalty cost of shortage allotment at the end of the planning horizon is not linear because the acquisition cost incurred by the backlogged shipment may spread over a few periods. Again we use a convex increasing function to reflect this. The policy is restricted to a single stage decision, i.e., to acquire allotment only for the next immediate departure.

In the following convexity the analysis is presented that the optimal return function in task (1) with respect to the backlogged shipment is a convex increasing function. Given this nice property, the task (1) can be efficiently solved recursively starting from the last stage. For the simplicity of presentation all functions are assumed to be differentiable.

Let $Z(v)$ be a non-negative convex increasing function of $v$ with $\lim _{v \rightarrow \infty} Z_{\mathrm{V}}=\infty$ and $Z(0)=0$, and $G(v)$ be a positive convex decreasing function of $v$ where the domain is restricted to $M^{+}$, the set of nonnegative real numbers. Define:

$$
Q(A)=\min _{\mathrm{r}}\{Z(r)+G(A+r)\} .
$$

Then $Q(A)$ is a convex decreasing function of $A$. Proof:

$Q(A)$ is well defined. i.e., $Q(A)$ has finite value for $A \in M^{+}$. This follows by observing that $Z(R)+G(a+R)$ is a convex function of $R$ and $\lim _{v \rightarrow \infty}$ $Z(v)=\infty$. To prove that $Q(A)$ is a convex decreasing function of $A$, it suffices to show that

$$
Q^{\prime}\left(A_{2}\right) \leq Q^{\prime}\left(A_{1}\right) \leq 0,
$$

whenever $A_{2} \leq A_{1}$.

Let the value $\tilde{A}$ be such that

$$
A^{\prime}(0)=-Z^{\prime}(\tilde{A})
$$

and

$$
Q(A)=Z\left(r^{*}\right)+G\left(A+r^{*}\right),
$$

where

$$
r^{*}=\min \{r: Q(A)=Z(r)+G(A+r)\},
$$

here for the simplicity of presentation we abuse the notation to let $B^{\prime}(0)$ represent the right-hand-side derivative of function $B$ at 0 . Similarly it applies to $G^{\prime}(\tilde{A})$ if $\tilde{A}$ happens to be 0 .

Case 1: $A \geq \tilde{A}$. It is not difficult to see that $r^{*}=$ 0 . Since $Q(A)=G(A)$ and $G(v)$ is a positive convex decreasing function of $v, Q^{\prime}\left(A_{2}\right) \leq Q^{\prime}\left(A_{1}\right) \leq 0$ is true for $\tilde{A} \leq A_{2} \leq A_{1}$.

Case 2: $A<\tilde{A}$. To show that $Q(A)$ is a convex decreasing function for the given range of $A$, we show that the function satisfies the following properties:

$$
\begin{aligned}
& A+r^{*} \leq \tilde{A}, \text { when, } A<\tilde{A}, \\
& A_{2}+r_{2}{ }^{*} \leq A_{1}+r_{1}{ }^{*} \leq \tilde{A} \text {, when, } A_{2} \leq A_{1} \leq \tilde{A}, \\
& Q^{\prime}\left(A_{2}\right) \leq Q^{\prime}\left(A_{1}\right) \leq Q^{\prime}(\widetilde{A}) \leq 0,
\end{aligned}
$$

whenever $A_{2} \leq A_{1} \leq \widetilde{A}$.

Let's prove (9). The optimal $r^{*}$ is such that

$$
Z^{\prime}\left(r^{*}\right)+G^{\prime}\left(A+r^{*}\right)=0 .
$$

This implies that

$$
-G^{\prime}\left(A+r^{*}\right)=Z^{\prime}\left(r^{*}\right) \geq Z^{\prime}(0)=-G(\widetilde{A})
$$

or

$$
-G^{\prime}\left(A+r^{*}\right) \leq G^{\prime}(\widetilde{A}) .
$$

As $G$ is a convex function, we have (9).

Let's prove (10). We first show that $r_{2}{ }^{*} \geq r_{1}{ }^{*}$. This follows by observing that $Z^{\prime}$ is an increasing function and

$$
-G^{\prime}\left(A_{2}+r_{1}^{*}\right) \geq-G^{\prime}\left(A_{1}+r_{1}^{*}\right)=Z^{\prime}\left(r_{1}\right) .
$$

Thus

$$
Z^{\prime}\left(r_{2}{ }^{*}\right)=-G^{\prime}\left(A_{2}+r_{2}{ }^{*}\right) \geq-G^{\prime}\left(A_{1}+r_{1}^{*}\right)=Z^{\prime}\left(r_{1}^{*}\right) \text {. }
$$

Next we show that $A_{2}+r_{2}{ }^{*} \leq A_{1}+r_{1}{ }^{*} \leq \widetilde{A}$. The in- 
equality $A_{1}+r_{1}{ }^{*} \leq \widetilde{A}$ is true because

$$
-G^{\prime}\left(A_{1}+r_{1}^{*}\right)=Z^{\prime}\left(r_{1}^{*}\right) \geq Z^{\prime}(0)=-G^{\prime}(\widetilde{A})
$$

The inequality $A_{2}+r_{2}{ }^{*} \leq A_{1}+r_{1}{ }^{*}$ is true because $G$ is a convex function and

$$
-G^{\prime}\left(A_{2}+r_{2}^{*}\right)=Z^{\prime}\left(r_{2}^{*}\right) \geq Z^{\prime}\left(r_{1}\right)=-G^{\prime}\left(A_{1}+r_{1}^{*}\right)
$$

Let's prove (11). This follows because

$$
\begin{aligned}
& \frac{d Q}{d A}=Z^{\prime}\left(r^{*}\right) \frac{d r^{*}}{d A}+G^{\prime} \cdot\left(A=r^{*}\right) \cdot\left(1+\frac{d r^{*}}{d A}\right)= \\
& Z^{\prime}\left(r^{*}\right) \frac{d r^{*}}{d A}+G^{\prime}\left(A=r^{*}\right) \frac{d r^{*}}{d A}+G^{\prime}\left(A+r^{*}\right)= \\
& {\left[Z^{\prime}\left(r^{*}\right)+G^{\prime}\left(A+r^{*}\right)\right] \frac{d r^{*}}{d A}+G^{\prime}\left(A+r^{*}\right)=G^{\prime}\left(A+r^{*}\right) \leq 0 .}
\end{aligned}
$$

From (10) we have

$$
\begin{aligned}
& Q^{\prime}\left(A_{2}\right)=G^{\prime}\left(A_{2}+r_{2}{ }^{*}\right) \leq G^{\prime}\left(A_{1}+r_{1}^{*}\right)= \\
& =Q^{\prime}\left(A_{1}\right) \leq G^{\prime}(\widetilde{A})=Q^{\prime}(\widetilde{A}) \leq 0 .
\end{aligned}
$$

The optimal return $Q_{i}$ function at stage $i$, $i=1, \ldots, N$ for task 1 is a convex increasing function with respect to the backlogged shipment, $B_{i-1}$. Let's prove it by induction. Consider the last stage, $i=N$. We have $Q_{N}\left(B_{N-1}\right)=L\left(B_{N-1}\right)$. Since $L$ is an increasing convex function, $Q_{N}$ is a convex increasing function with respect to $\mathrm{B}_{N-1}$. Consider the expected cost at stage $m$. Given $Q_{m+1}$ is a convex increasing function of $B_{m}$, then

$$
\begin{aligned}
& Q_{m} \cdot\left(B_{m-1}\right)=C \cdot B_{m-1}+\min _{R_{m}}\left\{Z\left(R_{m}\right)+\right. \\
& \left.E_{W_{m}}\left[\max \left\{Q_{m+1} \cdot\left(W_{m}-\left(A_{m}-B_{m-1}+R_{m}\right)\right), Q_{m+1}(0)\right\}\right]\right\} .
\end{aligned}
$$

\section{Define}

$$
\begin{aligned}
& G\left(A_{m}-B_{m-1}+R_{m}\right)= \\
& \left.\quad E_{W_{m}}\left[\max \left\{Q_{m+1} \cdot\left(W_{m}-\left(A_{m}-B_{m-1}+R_{m}\right)\right), Q_{m+1}(0)\right\}\right]\right\}
\end{aligned}
$$

and

$$
Q\left(A_{m}-B_{m-1}\right)=\min _{R_{m}}\left\{Z\left(R_{m}\right)+G\left(A_{m}-B_{m-1}+R_{m}\right)\right\}
$$

Then $G$ is a convex decreasing function of $A_{m}-B_{m-1}+R_{m}$ since both MAX operator and the expected value operator preserve convexity.

We can easily see that $Q_{m}\left(B_{m-1}\right)=B_{m-1}+Q\left(A_{m}-\right.$ $\left.B_{m-1}\right)$ is also a convex increasing function of $B_{m-1}$.

\section{Conclusions}

1. In this paper a model and a solution procedure for solving a problem of allotment in a shuttle train for transport units are presented. Forwarders should balance the cost of late deliveries against the cost of having too much allotment.

2. This model could be applicable in the growing market of trucks and containers transportation by shuttle trains in East Europe, where forwarders meet trying to manage the uncertainty of demand with the early booked allotment in a shuttle train.

\section{References}

1. Jaržemskis A. The perspectives of a shuttle-train concept. In: Proceedings of the international conference "Transport means-2002" (Transporto priemonès-2002: tarptautinès konferencijos pranešimų medžiaga). Kaunas: Technologija, 2002, p. 76-80 (in Lithuanian).

2. Chew, E. P.; Huang, H. C.; Johnson, E. L.; Nemhauser, G. L.; Sokol, J.; Leong, C. H. Short-term booking of air cargo space. Technical Report. Atlanta: Georgia Institute of Technology. $14 \mathrm{p}$.

3. Raman, A.; Tayur, S.; Ganeshan, R.; Magazine, M. Managing inventory for fashion products. Quantitative models for supply chain management. Norwell: Kluwer Academic Publishers, 1999, p. 789-805.

4. Ibaraki, T.; Imahori, S.; Kubo, M.; Masuda, T.; Uno, T.; Yagiura, M. Effective Local Search Algorithms for Routing and Scheduling Problems with General Time-Window Constraints. Transportation Science, Maryland: Institute for Operation Research and Management Sciences, Vol 39, Issue 2, 2005, p. 206232. ISSN 0041-1655.

5. Wu, P.; Hartman, J. C.; Wilson, G. R. An Integrated Model and Solution Approach for Fleet Sizing with Heterogeneous Assets. Transportation Science, Maryland: Institute for Operation Research and Management Sciences, Vol 39, Issue 1, 2005, p. 87-103. ISSN: 0041-1655. 